\title{
A Note on the Elliptic Sine-Gordon Equation
}

\author{
Goong Chen, Zhonghai Ding, Chia-Ren Hu, Wei-Ming Ni, and Jianxin \\ Zhou
}

\begin{abstract}
The elliptic sine-Gordon equation originates from the static case of the hyperbolic sine-Gordon equation modeling the Josephson junction in superconductivity. However, the elliptic sine-Gordon boundary value problem as studied in the mathematical literature actually has an opposite sign in front of the sine nonlinearity; it models not the "usual" Josephson junction but rather the Josephson $\pi$-junction, which is of contemporary interest to physicists. We first furnish this physical backdrop that has motivated our study here. Then we aim to establish the existence of nonconstant solutions of the semilinear elliptic sine-Gordon equation subject to homogeneous Neumann and Dirichlet boundary conditions by using critical point theory. Positive numerical solutions of the Dirichlet case, which are global minima of the variational problem, are computed on a dumbbell-shaped $2 \mathrm{D}$ domain for visualization.
\end{abstract}

\section{Origin of the model}

The hyperbolic sine-Gordon equation

$$
\phi_{x x}-\phi_{t t}=\sin \phi
$$

describes the dynamics of many condensed matter systems. Examples include: a 1-D ferromagnet with planar anisotropy in the presence of a magnetic field perpendicular to the chain direction [Mi], spin dynamics of the A-phase of superfluid ${ }^{3} \mathrm{He}$ $[\mathbf{M K}]$, and a Josephson transmission line [Sc], etc., besides such classical examples as: a chain of coupled pendula [Dr], and a classical model on 1-D dislocation [La].

The sine-Gordon equation may be derived from the Lagrangian:

$$
\mathcal{L}=\int d x\left(\frac{1}{2} \phi_{t}^{2}-\frac{1}{2} \phi_{x}^{2}+\cos \phi-1\right)
$$

2000 Mathematics Subject Classification. Primary: 35J20, 58E05; Secondary: 35Q20, $65 \mathrm{~N} 30$.

Key words and phrases. Elliptic sine-Gordon equation, variational methods, numerical computation.

G. Chen was supported in part by a TITF initiative from Texas A\&M University, C. R. $\mathrm{Hu}$ was supported in part by a grant from Texas Center for Superconductivity and Advanced Materials at the University of Houston, and W. M. Ni was supported in part by NSF Grant DMS 9988635 .

(C)0000 (copyright holder) 
or from the Hamiltonian:

$$
\mathcal{H}=\int d x\left(\frac{1}{2} \phi_{t}^{2}+\frac{1}{2} \phi_{x}^{2}+1-\cos \phi\right) .
$$

In the case of the Josephson transmission line, the variable $\phi$ above describes the relative phase, $\phi=\phi_{I}-\phi_{I I}$, between the superconducting metals $I$ and $I I$, which causes the Josephson tunneling current to flow across a very thin insulating barrier. If the Josephson transmission line is wide as well as long, the hyperbolic sine-Gordon equation must be generalized to two space dimensions and one in time:

$$
\phi_{x x}+\phi_{y y}-\phi_{t t}=\sin \phi .
$$

A somewhat detailed derivation of (1.4) may be found in [RS](p. 30); see (1.6)(1.10) below. The static version of this equation reads

$$
\phi_{x x}+\phi_{y y}=\sin \phi
$$

cf.[RS] (p. 31, (1.88)).

Now, consider however, a Josephson $\pi$-junction, which is formed by either inserting a ferromagnetic layer of a sufficient thickness inside the tunneling barrier perpendicular to the current direction $[\mathbf{K u}]$, or using $d$-wave high- $\mathrm{T}_{\mathrm{c}}$ superconductors to form the two electrodes of the junction in an appropriate arrangement [SR]. Thus, the basic equations modeling the Josephson $\pi$-junction (based on the very recent papers $[\mathbf{K u}]$ by T. Kontos et al. and Y. Blum et al.) now become

$$
\begin{aligned}
\frac{\partial \phi}{\partial t} & =\frac{2 e V}{\hbar}, \\
\frac{\partial \phi}{\partial x} & =\left(\frac{2 e d}{\hbar c}\right) H_{2}, \\
\frac{\partial \phi}{\partial y} & =\left(-\frac{2 e d}{\hbar c}\right) H_{1}, \\
j_{3} & =-j_{0}(x, y) \sin \phi,
\end{aligned}
$$

where

$$
\begin{aligned}
& e=\text { electron charge; } c=\text { the speed of light in vacuo; } \\
& d=\lambda_{1}+\lambda_{2}+b, \text { with } \\
& \lambda_{1}, \lambda_{2} \text { equal to, respectively, the London penetration depths for } \\
& \text { metals } I \text { and } I I \text {; } \\
& j_{3}(x, y)=\text { the Josephson current per unit area across the barrier, }
\end{aligned}
$$

$H_{1}, H_{2}$ are, respectively, the $x$ and $y$ components of the magnetic field;

$\hbar=$ Planck's constant; and

$V(x, y, t)=$ the potential difference across the barrier.

See Fig. 1.

Substituting (1.6)-(1.9) into the Maxwell equation [RS] (p. 30, (1.84))

$$
\frac{\partial H_{2}}{\partial x}-\frac{\partial H_{1}}{\partial y}-\frac{4 \pi}{4} \cdot C \cdot \frac{\partial V}{\partial t}=\frac{4 \pi}{c} j_{3}
$$




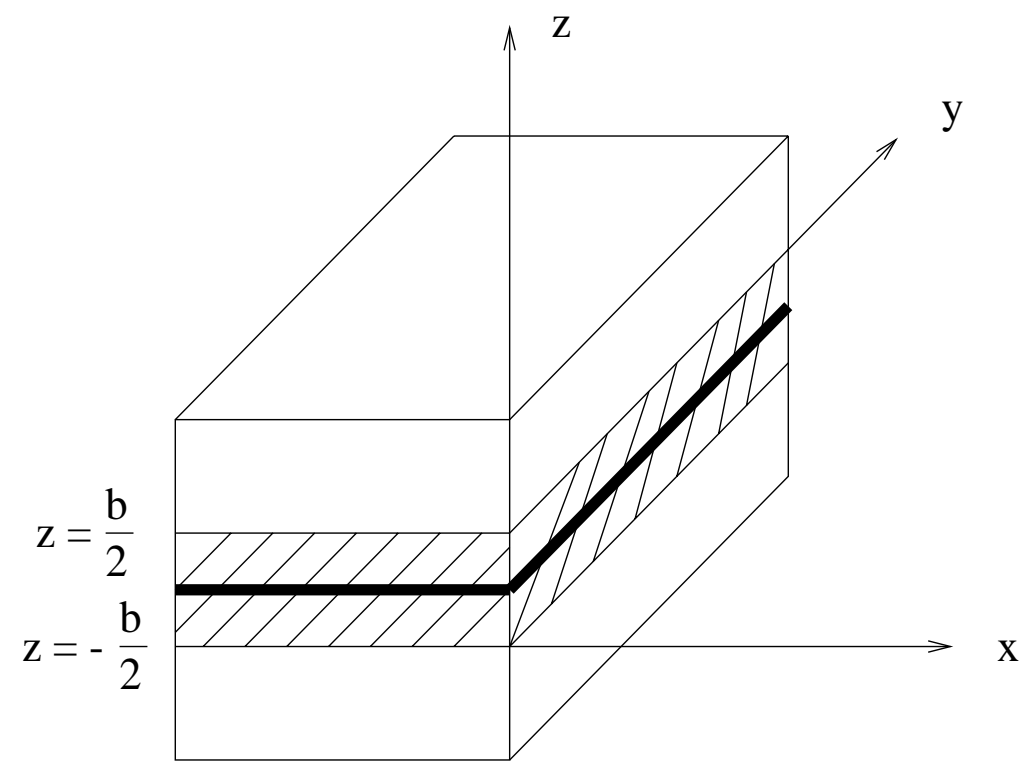

Figure 1. A Josephson $\pi$-junction. For $z>b / 2$, there is superconducting metal $I$ and for $z<-b / 2$, superconducting metal $I I$. The insulating barrier lying between $-b / 2<z<b / 2$ (the light shaded area) is inserted with a thin layer (the dark area) of ferromagnetic material, causing $j_{3}$ in (1.10) to have a negative sign. (This is the namesake for the $\pi$-junction as it means the change of phase by $\pi$, yielding a negative sign for $j_{3}$.)

yields the barrier equation

$$
\Delta \phi-\frac{1}{c_{0}^{2}} \frac{\partial^{2} \phi}{\partial t^{2}}=-\lambda_{J}^{-2} \sin \phi
$$

where $c_{0}=c(4 \pi C D)^{-1 / 2}, \lambda_{J}=c\left[\hbar /\left(8 \pi e d j_{0}\right)\right]^{1 / 2}, \Delta=\partial^{2} / \partial x^{2}+\partial^{2} / \partial y^{2}$, which, after normalization, gives

$$
\phi_{x x}+\phi_{y y}-\phi_{t t}=-\sin \phi,
$$

with static version

$$
\phi_{x x}+\phi_{y y}=-\sin \phi .
$$

This is the elliptic sine-Gordon equation we intend to investigate (rather than (1.5)) in this paper.

The boundary condition is the natural boundary condition

$$
\frac{\partial \phi}{\partial n}=0
$$

where $n$ is the unit outward normal vector on the boundary. In order to secure the Dirichlet boundary condition $\phi=0$ or the Robin condition $\phi+\alpha \partial \phi / \partial n=0$, it is apparent that some devices or controls must be imposed thereupon. We have not yet found much discussion about how to carry out the design of such devices or controls in the literature, however. 
In this paper, we present some theoretical and numerical results for the elliptic sine-Gordon boundary value problem. We will discuss both the homogeneous Dirichlet and Neumann boundary conditions, with the understanding that the discussion so far for the homogeneous Dirichlet condition is primarily of academic interest. Even though the existence of multiple solutions of a general class of nonlinear eigenvalue problems has already been discussed in [Ra] (in particular, Theorem 9.6), however, we must profess that for the elliptic sine-Gordon equation, the understanding of qualitative properties of the nontrivial solutions rather than the existence of multiple nontrivial solutions seems to be limited, at least as far as the authors are concerned. Furthermore, this paper should be viewed more as a research in progress rather than a major milestone on this subject.

\section{The elliptic sine-Gordon equation $(I)$ : Neumann boundary condition}

Let $\Omega$ be a bounded open domain in $\mathbb{R}^{N}$ with sufficiently smooth boundary $\partial \Omega$. Let $H^{s}(\Omega)$ denote the usual Sobolev space of order $s \in \mathbb{R}$. Consider the boundary value problem (BVP)

$$
\begin{cases}-\Delta w(x)=\lambda \sin w(x), & x=\left(x_{1}, \ldots, x_{N}\right) \in \Omega, \quad \lambda>0, \\ \frac{\partial w}{\partial n}=0 & \text { on } \partial \Omega .\end{cases}
$$

The variational functional corresponding to (2.1) is

$$
J(v)=\int_{\Omega}\left[\frac{1}{2}|\nabla v|^{2}-\lambda(1-\cos v)\right] d x, \quad v \in H^{1}(\Omega) .
$$

Sometimes, for clarity we will write $J$ as $J_{\lambda}$ to signify its dependence on $\lambda$.

REMARK 2.1. By making the change of variable

$$
w(x)=\pi-v(x), \quad x \in \Omega,
$$

in (2.1), we obtain

$$
\begin{cases}-\Delta v(x)=-\lambda \sin v(x), & x \in \Omega, \\ \frac{\partial v}{\partial n}=0 & \text { on } \Omega .\end{cases}
$$

Thus, we see that as far as the Neumann BVP is concerned, mathematically speaking, the Josephson $\pi$-function and the usual Josephson junction are equivalent.

However, for the homogeneous Dirichlet BVP, the change of variable (2.3) is inadmissible, and thus the equations for the Josephson $\pi$-junction and the usual Josephson junction are not equivalent.

Using the calculus of variations, one can verify that if $w_{0} \in H^{1}(\Omega)$ is a critical point of $J$, i.e., $J^{\prime}\left(w_{0}\right)=0$, then $w_{0} \in H^{2}(\Omega)$ and $w_{0}$ is a classical solution of (2.1), and vice versa that if $w_{0}$ satisfies $(2.1)$, then $w_{0}$ is a critical point of $J$ satisfying $J^{\prime}\left(w_{0}\right)=0$.

LEMma 2.2. $J(v)$ has a local minimum at $v=\pi$ and, consequently, at every $(2 n-1) \pi$ for every $n=0, \pm 1, \pm 2, \ldots$.

Proof. Let $\varepsilon>0$. For $v_{0} \in H^{1}(\Omega)$, define

$$
B\left(v_{0}, \varepsilon\right)=\left\{v \in H^{1}(\Omega) \mid\left\|v-v_{0}\right\|_{H^{1}(\Omega)}<\varepsilon\right\} .
$$


For $v \in \partial B(0, \varepsilon)$, we have

$$
\begin{aligned}
J(\pi+v)-J(\pi) & =\left\{\frac{1}{2} \int_{\Omega}|\nabla v|^{2} d x-\lambda \int_{\Omega}[1-\cos (\pi+v)] d x\right\}+2 \lambda \int_{\Omega} d x \\
& =\frac{1}{2} \int_{\Omega}|\nabla v|^{2} d x+\lambda \int_{\Omega}(1-\cos v) d x \\
& =\frac{1}{2} \int_{\Omega}|\nabla v|^{2} d x+\lambda \int_{\Omega}\left[\frac{v^{2}}{2 !}-\frac{v^{4}}{4 !}+\frac{v^{6}}{6 !}-\cdots\right] d x \\
& \geq \frac{1}{2} \int_{\Omega}|\nabla v|^{2} d x+\frac{\lambda}{4} \int_{\Omega} v^{2} d x,
\end{aligned}
$$

if $\varepsilon<\varepsilon_{0}$ for some small $\varepsilon_{0}>0$. Thus

$$
J(\pi+v)-J(\pi) \geq \min \left\{\frac{1}{2}, \frac{\lambda}{4}\right\}\|v\|_{H^{1}(\Omega)}^{2} .
$$

Hence

$$
J(v)-J(\pi) \geq \delta_{0}, \text { for some } \delta_{0}>0 \text { for all } v \in \partial B(\pi, \varepsilon) .
$$

Let $\left\{\lambda_{j} \mid 0=\lambda_{1}<\lambda_{2}<\cdots<\lambda_{n}<\cdots, j=1,2, \ldots\right\}$ be the set of eigenvalues of the Neumann boundary value problem

$$
\begin{cases}-\Delta \phi_{j}=\lambda_{j} \phi_{j} & \text { on } \Omega, \\ \frac{\partial \phi_{j}}{\partial n}=0 & \text { on } \partial \Omega .\end{cases}
$$

THEOREM 2.3. If $\lambda>\lambda_{2}$, then $J_{\lambda}(v)$ has at least two nonconstant critical points $w$ of mountain-pass type in $H^{1}(\Omega)$. Consequently, $w(x)+2 k \pi$ are also solutions of (2.1) for any integer $k$.

Proof. Since $J \in C^{1}\left(H^{1}(\Omega), \mathbb{R}\right)$ and it is straightforward to verify that $J$ satisfies the Palais-Smale (PS) condition, by Lemma 2.2 and the Mountain Pass Lemma $[\mathbf{A R}]$, we see that $J(v)$ has at least a critical point $w_{0} \in H^{1}(\Omega)$ such that

$$
J\left(w_{0}\right)=\inf _{\gamma \in \Gamma} \sup _{v \in \gamma([0,1])} J(v)>\delta_{0}>0,
$$

where $\Gamma=\left\{\gamma \in C\left(\mathbb{R}, H^{1}(\Omega)\right) \mid \gamma(0)=\pi, \gamma(1)=-\pi\right\}$.

Next, we prove that if $\lambda>\lambda_{2}$, then $w_{0} \not \equiv 0$. For $\phi_{2}$ satisfying (2.5), we have $\int_{\Omega} \phi_{2} d x=0$. If $\lambda>\lambda_{2}$, then

$$
\begin{aligned}
J\left(t \phi_{2}\right) & =\frac{t^{2}}{2} \int_{\Omega}\left|\nabla \phi_{2}\right|^{2} d x-\lambda \int_{\Omega}\left(1-\cos \left(t \phi_{2}\right)\right) d x \\
& =\frac{\lambda_{2} t^{2}}{2} \int_{\Omega} \phi_{2}^{2} d x-\lambda \int_{\Omega}\left[\frac{t^{2} \phi_{2}^{2}}{2 !}-\frac{t^{4} \phi_{2}^{4}}{4 !}+\frac{t^{6} \phi_{2}^{6}}{6 !}-\cdots\right] d x \\
& =t^{2}\left\{\frac{\left(\lambda_{2}-\lambda\right)}{2} \int_{\Omega} \phi_{2}^{2} d x+t^{2} \lambda \int_{\Omega}\left[\frac{\phi_{2}^{4}}{4 !}-\frac{t^{2} \phi_{2}^{6}}{6 !}+\cdots\right] d x\right\} .
\end{aligned}
$$


Thus

$$
<J^{\prime \prime}(0) \phi_{2}, \phi_{2}>_{H^{1}}=\left(\lambda_{2}-\lambda\right) \int_{\Omega} \phi_{2}^{2} d x<0 .
$$

Note that $\phi_{1}=1$. From the definition of $J$ in (2.2), one can verify easily that

$$
<J^{\prime \prime}(0) \phi_{1}, \phi_{1}>_{H^{1}}=-\lambda|\Omega|<0 .
$$

Note that $\phi_{1}$ and $\phi_{2}$ are perpendicular in $H^{1}(\Omega)$. Thus the Morse index of $J$ at $w=0$, which is defined by the dimension of the maximal subspace of $H^{1}(\Omega)$ where $J^{\prime \prime}(0)$ is negative definite $[\mathbf{C h}]$, is greater than or equal to 2 . However, since $w_{0}$ is a critical point of $J$ of Mountain-Pass type, the Morse index of $J$ at $w_{0}$ is less than or equal to $1[\mathbf{C h}]$. Thus, $w_{0} \not \equiv 0$. Therefore, $J_{\lambda}(v)$ has two critical points, $\pm w_{0}$, of the mountain pass type, if $\lambda>\lambda_{2}$.

By using a different approach based upon the Mountain Pass Lemma in order intervals $[\mathbf{L W}], \mathrm{C}$. Li has studied a class of nonlinear elliptic equations similar to the elliptic sine-Gordon equation in a recent preprint $[\mathbf{L i}]$. By applying Theorem 1.1 in [Li] , (2.1) admits two nonconstant solutions between $-\pi$ and $\pi$ if $\lambda>\lambda_{2}$. However, it is unclear whether those two solutions are of mountain-pass type as what we claimed in Theorem 2.3. Our proof here is somewhat more elementary and easier to understand.

The following theorem gives us some additional information about the bounds of the solutions of (2.1).

THEOREM 2.4. The BVP (2.1) does not admit any nonconstant solution $w$ such that $0<w(x)<\pi$ on $\Omega$, for any $\lambda>0$. Consequently, for any $\lambda>0,(2.1)$ does not admit any nonconstant solution $w$ such that

$$
2 n \pi<w(x)<(2 n+1) \pi,
$$

for any integer $n$.

Proof. Assume that (2.1) admits a nonconstant solution $w(x)$ such that $0<$ $w(x)<\pi$, then one can easily check $v(x)=\pi-w(x)$ is a solution of (2.4). Multiplying the first equation of (2.4) by $v$ and integrate over $\Omega$, we have

$$
-\int_{\Omega}|\nabla v|^{2} d x=\lambda \int_{\Omega} v \sin v d x \geq 0
$$

Thus

$$
\int_{\Omega}|\nabla v|^{2} d x=0, \quad \text { and } \quad \int_{\Omega} v \sin v d x=0
$$

implying $v \equiv 0$ or $v \equiv \pi$, a contradiction.

\section{The elliptic sine-Gordon equation (II): Dirichlet boundary condition}

For the Dirichlet BVP

$$
\begin{cases}\Delta w+\lambda \sin w=0 & \text { on } \Omega \\ w=0 & \text { on } \partial \Omega,\end{cases}
$$

the variational functional $J$ remains the same as in (2.2), but the underlying Hilbert space becomes $H_{0}^{1}(\Omega)=\left\{w \in H^{1}(\Omega) \mid w=0\right.$ on $\left.\partial \Omega\right\}$. We let $\left\{\tilde{\lambda}_{j} \mid 0<\tilde{\lambda}_{1}<\tilde{\lambda}_{2}<\right.$ 
$\left.\cdots<\tilde{\lambda}_{n}<\cdots, j=1,2,3, \ldots\right\}$ denote the set of eigenvalues of the homogeneous Dirichlet BVP

$$
\begin{cases}-\Delta \tilde{\phi}_{n}=\tilde{\lambda}_{n} \tilde{\phi}_{n}, & \text { on } \Omega \\ \tilde{\phi}_{n}=0 & \text { on } \partial \Omega .\end{cases}
$$

It is straightforward to verify that $J(0)=0, J \in C\left(H_{0}^{1}(\Omega), \mathbb{R}\right)$ and that solutions $w$ of (3.1) correspond exactly to critical points $w$ of $J$ satisfying $J^{\prime}(w)=0$.

Proposition 3.1. Let $\lambda$ satisfy $0<\lambda<\tilde{\lambda}_{1}$. Then $w \equiv 0$ is the only critical point of $J_{\lambda}$.

Proof. Assume $w \in H_{0}^{1}(\Omega)$ is a critical point of $J$ and $w \neq 0$. Then $w$ is a weak solution of (3.1). By multiplying (3.1) by $w$ and integrating over $\Omega$, one has

$$
0<\int_{\Omega}|\nabla w|^{2} d x=\lambda \int_{\Omega} w \sin w d x \leq \lambda \int_{\Omega} w^{2} d x .
$$

By the Poincaré inequality, one then has

$$
0<\int_{\Omega}|\nabla w|^{2} d x \leq \frac{\lambda}{\tilde{\lambda}_{1}} \int_{\Omega}|\nabla w|^{2} d x .
$$

Thus $\lambda \geq \tilde{\lambda}_{1}$ which contradicts to the assumption $0<\lambda<\tilde{\lambda}_{1}$. Therefore $w=0$ is the only critical point of $J_{\lambda}$ if $0<\lambda<\tilde{\lambda}_{1}$.

For any $v \in H_{0}^{1}(\Omega)$, we have

$$
J(v)=\int_{\Omega}\left[\frac{1}{2}|\nabla v|^{2}-\lambda(1-\cos v)\right] d x \geq \frac{1}{2} \int_{\Omega}|\nabla v|^{2} d x-2 \lambda|\Omega|,
$$

where $|\Omega|$ denotes the Lebesgue measure of $\Omega$. Also, for any $v \in H_{0}^{1}(\Omega), v \neq 0$, $J(t v)$ is continuous with respect to $t \in \mathbb{R}$ and

$$
\lim _{t \rightarrow \pm \infty} J(t v)=\infty
$$

So $\min _{t \in \mathbb{R}} J(t v)$ exists and there is a $t^{*} \in \mathbb{R}$ such that

$$
J\left(t^{*} v\right)=\min _{t \in \mathbb{R}} J(t v) .
$$

We further deduce that

$$
m \equiv \inf _{v \in H_{0}^{1}(\Omega)} \min _{t \in \mathbb{R}} J(t v)=\min _{v \in H_{0}^{1}(\Omega)} \min _{t \in \mathbb{R}} J(t v) \geq-2 \lambda|\Omega| .
$$

Let $\left\{v_{n}^{*} \in H_{0}^{1}(\Omega) \mid n=1,2,3, \ldots\right\}$ be such that

$$
\begin{gathered}
J\left(v_{n}^{*}\right)=\min _{t \in \mathbb{R}} J\left(t v_{n}^{*}\right), \\
\lim _{n \rightarrow \infty} J\left(v_{n}^{*}\right)=m .
\end{gathered}
$$

Then, since

$$
J\left(v_{n}^{*}\right) \geq \frac{1}{2} \int_{\Omega}\left|\nabla v_{n}^{*}\right|^{2} d x-2 \lambda|\Omega|,
$$

we deduce that $\left\{v_{n}^{*} \mid n=1,2,3, \ldots\right\}$ is bounded in $H_{0}^{1}(\Omega)$. Thus $\left\{v_{n}^{*}\right\}$ has a weakly convergent subsequence, still denoted the same, such that

$$
\lim _{n \rightarrow \infty} v_{n}^{*}=v_{0}^{*} \quad \text { weakly in } \quad H_{0}^{1}(\Omega) .
$$


Since $J$ is continuously Fréchet differentiable, we have

$$
m \leq J\left(v_{0}^{*}\right) \leq \lim _{n \rightarrow \infty} J\left(v_{n}^{*}\right)=m=\min _{v \in H_{0}^{1}(\Omega)} J(v) .
$$

We summarize the above in the following.

Lemma 3.2. Let $\lambda>0$. Then $J_{\lambda}(\cdot)$ has a global minimizer $v_{0}^{*}$ in $H_{0}^{1}(\Omega)$ satisfying (3.1). If $v_{0}^{*} \not \equiv 0$, then $-v_{0}^{*}$ is also a global minimizer.

THEOREM 3.3. If $\lambda>\tilde{\lambda}_{1}$, then the global minimizer $v_{0}^{*}$ in Lemma 3.2 is nontrivial: $v^{*} \not \equiv 0$. Therefore, $-v_{0}^{*}$ is also a distinct global minimizer.

Proof. We need only to prove that there is a $v_{0} \neq 0, v_{0} \in H_{0}^{1}(\Omega)$ such that $J\left(v_{0}\right)<0=J(0)$.

Let $v_{0}=t \tilde{\phi}_{1}$, cf. (3.2). Then

$$
\begin{aligned}
J\left(t \tilde{\phi}_{1}\right) & =\frac{t^{2}}{2} \int_{\Omega}\left|\nabla \tilde{\phi}_{1}\right|^{2} d x-\lambda \int_{\Omega}\left(1-\cos \left(t \tilde{\phi}_{1}\right)\right) d x \\
& =\frac{\tilde{\lambda}_{1} t^{2}}{2} \int_{\Omega}\left|\tilde{\phi}_{1}\right|^{2} d x-\lambda \int_{\Omega}\left(1-\cos \left(t \tilde{\phi}_{1}\right)\right) d x .
\end{aligned}
$$

Using

$$
\cos x=1-\frac{x^{2}}{2 !}+\frac{x^{4}}{4 !} \cdots \cdots, \quad x \in \mathbb{R}
$$

we have

$$
J\left(t \tilde{\phi}_{1}\right)=\frac{1}{2}\left(\tilde{\lambda}_{1}-\lambda\right) t^{2} \int_{\Omega}\left|\tilde{\phi}_{1}\right|^{2} d x+\lambda \int_{\Omega}\left[\frac{t^{4}\left|\tilde{\phi}_{1}\right|^{4}}{4 !}-\cdots\right] d x .
$$

Since $\tilde{\phi}_{1}$ is $C^{\infty}$ on $\Omega,\left\|\tilde{\phi}_{1}\right\|_{L^{\infty}(\Omega)}<+\infty$, and

$$
\begin{aligned}
J\left(t \tilde{\phi}_{1}\right) & =t^{2}\left\{-\frac{1}{2}\left(\lambda-\tilde{\lambda}_{1}\right) \int_{\Omega}\left|\tilde{\phi}_{1}\right|^{2} d x+\lambda t^{2} \int_{\Omega}\left[\frac{\left|\tilde{\phi}_{1}\right|^{4}}{4 !}-\frac{t^{2}\left|\tilde{\phi}_{1}\right|^{6}}{6 !}+\cdots\right] d x\right\} \\
& \equiv t^{2}\left\{T_{1}+\lambda t^{2} T_{2}\right\},
\end{aligned}
$$

where $T_{1}$ is a negative constant, and $T_{2}$ depends on $t$ such that for $|t|$ small, $T_{2}$ is positive.

Thus, when $t$ is small enough, i.e., there exists $\delta_{0}>0$ such that

$$
J\left(t \tilde{\phi}_{1}\right)<0 \text { if }|t|<\delta_{0} .
$$

So the proof is complete.

THEOREM 3.4. Let $\lambda>\tilde{\lambda}_{1}$. If $w_{0}$ is global minimizer of $J$, then $w_{0}$ is of one sign.

Proof. Note that $w_{0}$ is a classical solution of (3.1), and $w_{0} \in H^{2}(\Omega) \cap H_{0}^{1}(\Omega)$ by regularity theory. Write

$$
w_{0}(x)=w_{0}^{+}(x)+w_{0}^{-}(x) ; \quad w_{0}^{+}=\max \left(w_{0}, 0\right), \quad w_{0}^{-}=\min \left(w_{0}, 0\right) .
$$

Thus $w_{0}^{+} \in H_{0}^{1}(\Omega), w_{0}^{-} \in H_{0}^{1}(\Omega)$. It is easy to check that

$$
J\left(w_{0}\right)=J\left(w_{0}^{+}\right)+J\left(w_{0}^{-}\right) .
$$


If $w_{0}$ changes signs on $\Omega$, then

$$
J\left(w_{0}^{+}\right)<J\left(w_{0}\right), \quad J\left(w_{0}^{-}\right)<J\left(w_{0}\right),
$$

contradicting that $w_{0}$ is a global minimizer of $J$ on $H_{0}^{1}(\Omega)$. Thus either $w_{0}=w_{0}^{+}$ or $w=w^{-}$.

Theorem 3.5. Let $\lambda>\tilde{\lambda}_{1}$. If $w_{0}(x) \geq 0$ is the global minimizer of $J$ on $H_{0}^{1}(\Omega)$, then $0<w_{0}(x)<\pi$ on $\Omega$.

Proof. Assume $w_{0}(x) \geq 0$ is the global minimizer of $J$ on $H_{0}^{1}(\Omega)$, and $\max _{x \in \Omega} w_{0}(x)$ $>\pi$. Thus, define

$$
w_{\pi}(x)=\left\{\begin{array}{lll}
\pi, & \text { if } \quad w_{0}(x) \geq \pi \\
w_{0}(x), & \text { if } \quad w_{0}(x)<\pi .
\end{array}\right.
$$

We have $w_{\pi} \in H_{0}^{1}(\Omega)$, and

$$
\begin{aligned}
J\left(w_{0}\right)-J\left(w_{\pi}\right)= & {\left[\frac{1}{2} \int_{\Omega}\left|\nabla w_{0}\right|^{2} d x-\lambda \int_{\Omega}\left(1-\cos w_{0}\right) d x\right] } \\
& -\left[\frac{1}{2} \int_{\Omega}\left|\nabla w_{\pi}\right|^{2} d x-\lambda \int_{\Omega}\left(1-\cos w_{\pi}\right) d x\right] \\
= & \frac{1}{2} \int_{\Omega^{*}}\left|\nabla w_{0}\right|^{2} d x-\lambda \int_{\Omega^{*}}\left(\cos w_{\pi}-\cos w_{0}\right) d x
\end{aligned}
$$

where $\Omega^{*}=\left\{w \in \Omega \mid w_{0}(x) \geq \pi\right\}$, giving

$$
\begin{aligned}
J\left(w_{0}\right)-J\left(w_{\pi}\right) & =\frac{1}{2} \int_{\Omega^{*}}\left|\nabla w_{0}\right|^{2} d x-\lambda \int_{\Omega^{*}}\left(-1-\cos w_{0}\right) d x \\
& =\frac{1}{2} \int_{\Omega^{*}}\left|\nabla w_{0}\right|^{2} d x+\lambda \int_{\Omega^{*}}\left(1+\cos w_{0}\right) d x>0,
\end{aligned}
$$

if $w(x)$ crosses $\pi$, i.e., $\left|\Omega^{*}\right|>0$. This contradicts the fact that $w_{0}$ is a global minimizer of $J$ on $H_{0}^{1}(\Omega)$. Hence $0 \leq w_{0}(x) \leq \pi$. By a result of Ambrosetti and Hess $[\mathbf{A H}]$,

$$
\left\|w_{0}\right\|_{\infty} \neq \pi
$$

Thus $0 \leq w_{0}(x)<\pi$. By the maximum principle, we have $w_{0}(x)>0$ on $\Omega$. Therefore, $0<w_{0}(x)<\pi$ on $\Omega$.

Theorem 3.5 indicates that any global minimizer of $J_{\lambda}$ for $\lambda>\tilde{\lambda}_{1}$ is naturally positive on $\Omega$. In the following, we provide a few additional theorems on such a "positive solution."

Theorem 3.6. Let $\lambda>\tilde{\lambda}_{1}$. Assume that $w_{0}>0$ is a global minimizer of $J$ on $H_{0}^{1}(\Omega)$. Then $w_{0}$ is a maximal solution of (3.1) in the sense that if $w>0$ also satisfies (3.1) and $\|w\|_{\infty}<\pi$, then $w \leq w_{0}$ on $\Omega$, where \|\|$_{\infty}$ denotes the $L^{\infty}(\Omega)$-norm. 
Proof. From the proof of Theorem 3.5, we know that $\left\|w_{0}\right\|_{\infty}<\pi$. Rewrite (3.1) as

$$
\begin{cases}\left(-\Delta+\lambda_{0}\right) w_{0}=\lambda_{0} w_{0}+\lambda \sin w_{0}, & \text { on } \Omega \\ \left.w_{0}\right|_{\partial \Omega}=0 ; \quad w_{0}>0 & \text { on } \Omega\end{cases}
$$

where $\lambda_{0}>\lambda$ is a given number. Define $\mathcal{A}_{\lambda_{0}}=-\Delta+\lambda_{0}$ on $H_{0}^{1}(\Omega) \cap H^{2}(\Omega)$, and $f(u)=\lambda_{0} u+\lambda \sin u$. One can easily check that the following holds:

(1) $\sigma\left(\mathcal{A}_{\lambda_{0}}\right)=\left\{\lambda_{0}+\tilde{\lambda}_{n} \mid n=1,2,3, \ldots\right.$, cf. $\left.(3.2)\right\}$, where $\sigma$ denotes the spectrum;

(2) $f(u)$ is monotonically increasing, i.e., if $u<v, f(u)<f(v)$.

Let $\bar{w}_{0}=\pi$ on $\Omega$, then $\bar{w}_{0}$ is an upper solution of (3.7), while $w_{0}$ can be viewed as an lower solution of (3.7). Thus define

$$
\bar{w}_{n+1}=\mathcal{A}_{\lambda_{0}}^{-1} f\left(\bar{w}_{n}\right) .
$$

Then $\left\{\bar{w}_{n}\right\}$ is a monotone sequence such that

$$
w_{0} \leq \bar{w}_{n+1} \leq \bar{w}_{n} \leq \cdots \leq \bar{w}_{0}=\pi .
$$

Thus by the Monotone Iteration Theorem, there is a $\bar{w} \in H_{0}^{1}(\Omega) \cap C^{\infty}(\Omega)$ such that

$$
\lim _{n \rightarrow \infty} \bar{w}_{n}=\bar{w} \text {. }
$$

Thus $\bar{w}$ is a solution of (3.7), and

$$
w_{0} \leq \bar{w} \quad \text { on } \quad \Omega ;
$$

$\bar{w}$ is a maximal solution of (3.7) on $\Omega$ satisfying $\|\bar{w}\|_{\infty}<\pi$. Next, we show $w_{0} \equiv \bar{w}$. Note that

$$
\begin{aligned}
J\left(w_{0}\right) & =\frac{1}{2} \int_{\Omega}\left|\nabla w_{0}\right|^{2} d x-\lambda \int_{\Omega}\left(1-\cos w_{0}\right) d x \\
& =-\frac{1}{2} \int_{\Omega} \Delta w_{0} \cdot w_{0} d x-\lambda \int_{\Omega}\left(1-\cos w_{0}\right) d x \\
& =\frac{1}{2} \int_{\Omega} \lambda \sin w_{0} \cdot w_{0} d x-\lambda \int_{\Omega}\left(1-\cos w_{0}\right) d x \\
& =\lambda \int_{\Omega}\left[\frac{1}{2} w_{0} \sin w_{0}-\left(1-\cos w_{0}\right)\right] d x .
\end{aligned}
$$

Similarly,

$$
J(\bar{w})=\lambda \int_{\Omega}\left[\frac{1}{2} \bar{w} \sin \bar{w}-(1-\cos \bar{w})\right] d x .
$$

Note that $g(x)=\frac{1}{2} x \sin x-(1-\cos x)$ is monotonically decreasing on $[0, \pi]$ and, thus, $w_{0} \leq \bar{w}$ implies

$$
J\left(w_{0}\right) \geq J(\bar{w}) .
$$

But $w_{0}$ is a global minimizer of $J$. We thus conclude

$$
w_{0} \equiv \bar{w} \quad \text { on } \Omega .
$$

Therefore, $w_{0}$ is a maximal solution in the sense as stated in the theorem. 
Corollary 3.7. Let $\lambda>\tilde{\lambda}_{1}$. Let $w_{0}$ be a maximal solution satisfying the assumptions of Theorem 3.6. Assume that $w>0$ also satisfies (3.1) and $\|w\|_{\infty}<\pi$. Then $w \equiv w_{0}$ on $\Omega$, i.e., (3.1) has a unique positive solution satisfying $\|w\|_{\infty}<\pi$.

Proof. From Theorem 3.6, we have $w(x) \leq w_{0}(x), x \in \Omega$. Thus

$$
\begin{aligned}
0 & =\int_{\Omega}\left[\left(-\Delta w_{0}\right) w-(-\Delta w) w_{0}\right] d x \\
& =\int_{\Omega}\left[\lambda \sin w_{0} \cdot w-\lambda \sin w \cdot w_{0}\right] d x \\
& =\lambda \int_{\Omega} w w_{0}\left[\frac{\sin w_{0}}{w_{0}}-\frac{\sin w}{w}\right] d x
\end{aligned}
$$

Note that $w(x) w_{0}(x)>0$ on $\Omega$, and $\frac{\sin x}{x}$ is monotonically decreasing on $[0, \pi]$, thus,

$$
0=\lambda \int_{\Omega} w w_{0}\left[\frac{\sin w_{0}}{w_{0}}-\frac{\sin w}{w}\right] d x
$$

implying $w w_{0}\left[\frac{\sin w_{0}}{w_{0}}-\frac{\sin w}{w}\right] \equiv 0$ on $\Omega$ and hence $w=w_{0}$ on $\Omega$.

EXAMPLE 3.8 (Uniqueness of the positive solution on the disk). Let $\lambda>\tilde{\lambda}_{1}$. Consider

$$
\begin{cases}\Delta u=-\lambda \sin u & \text { on } \Omega, \\ u>0 & \text { on } \Omega, \\ u=0 & \text { on } \partial \Omega,\end{cases}
$$

where $\Omega=\left\{\left(x_{1}, x_{2}\right) \mid x_{1}^{2}+x_{2}^{2}<R^{2}\right\}$ for some $R>0$. By results in Gidas, Ni and Nirenberg [GNN], all solutions of (3.8) are radially symmetric. Thus, let

$$
w(r)=u(r, \theta) \text {; }
$$

$w(r)$ satisfies

$$
\begin{cases}\frac{d^{2} w}{d r^{2}}+\frac{1}{r} \frac{d w}{d r}=-\lambda \sin w, & 0 \leq r<R \\ w(r)>0, & 0 \leq r<R \\ w^{\prime}(0)=0, \quad w(R)=0 . & \end{cases}
$$

Multiply both sides of (3.9) by $\frac{d w}{d r}$, and integrate from 0 to $r$,

$$
\frac{1}{2}\left(\frac{d w}{d r}\right)^{2}+\int_{0}^{r} \frac{1}{r}\left(\frac{d w}{d r}\right)^{2} d r=\lambda(\cos w-\cos w(0)), \quad 0 \leq r<R .
$$

Since the left-hand side is always nonnegative, we must have $\cos w(0)=-1$ if $w(r)$ crosses $\pi$, in order to maintain the nonnegativity of the left-hand side. If $w(r)$ is a solution of (3.9) and $w(r)$ crosses $\pi$, then

$$
\frac{1}{2}\left(\frac{d w}{d r}\right)^{2}+\int_{0}^{r} \frac{1}{r}\left(\frac{d w}{d r}\right)^{2} d r=\lambda(1+\cos w) .
$$

If $w(r)$ crosses $\pi$ at $0<r_{0}<R$, i.e. $w\left(r_{0}\right)=\pi$, then

$$
\frac{1}{2}\left(\frac{d w}{d r}\right)^{2}\left(r_{0}\right)+\int_{0}^{r_{0}} \frac{1}{r}\left(\frac{d w}{d r}\right)^{2} d r=\lambda\left(1+\cos w\left(r_{0}\right)\right)=0 .
$$


Thus $\frac{d w}{d r}(r)=0,0 \leq r \leq r_{0}$. Then we deduce $w(r) \equiv \pi, 0 \leq r \leq r_{0}$. Therefore

$$
0<w(r) \leq \pi \quad \text { if } \quad 0 \leq r<R
$$

By $[\mathbf{A H}]$, we have

$$
\|w\|_{\infty} \not \equiv \pi .
$$

Thus, for $\lambda>\tilde{\lambda}_{1}$, a positive solution $w$ satisfies

$$
0<w(r)<\pi .
$$

Consequently, by Corollary (3.7), the positive solution is unique.

The preceding theorems and Example 3.8 all offer strong support to the conjecture that there is a unique positive solution to (3.1), if $\lambda>\tilde{\lambda}_{1}$. However, a complete proof is not available at this moment.

Sign-changing solutions of the elliptic sine-Gordon equation are of considerable significance because they induce the so-called Josephson vortex and, thus, corresponds to physical phenomena of practical interest. So let us consider sign-changing solutions of (3.1) in the rest of this section.

THEOREM 3.9. Let $\lambda>\tilde{\lambda}_{2}$. Then (3.1) admits at least four nontrivial solutions.

Proof. From Theorem 3.3, (3.1) admits at least two solutions $w_{0}$ and $-w_{0}$, where $w_{0} \in H_{0}^{1}(\Omega)$ is a global minimizer of $(2.2)$ on $H_{0}^{1}(\Omega)$, for $\lambda>\tilde{\lambda}_{1}$.

Since $J(v)$ satisfies the conditions in the Mountain Pass Lemma, and $w_{0}$ and $-w_{0}$ are two global minimizers of $J(v)$ with $J\left(w_{0}\right)=J\left(-w_{0}\right)$, we see that $J(v)$ has at least one critical point $w_{1}$ such that

$$
J\left(w_{1}\right)=\min _{\gamma \in \Gamma} \sup _{v \in \gamma([0,1])} J(v)>J\left(w_{0}\right),
$$

where

$$
\Gamma=\left\{\gamma \in C\left([0,1], H_{0}^{1}(\Omega)\right) \mid \gamma(0)=w_{0}, \gamma(1)=-w_{0}\right\} .
$$

The only thing left is to prove that $w_{1} \neq 0$.

Consider $J(v)$ near 0 , let $\tilde{\phi}_{2}$ be the eigenfunction of $-\Delta$ on $\Omega$ corresponding $\lambda=\tilde{\lambda}_{2}$ cf. (3.2). By repeating the same argument in the proof of Theorem 3.3, we have

$$
J\left(t \phi_{1}\right)=-\frac{t^{2}}{2}\left(\lambda-\tilde{\lambda}_{1}\right) \int_{\Omega}\left|\tilde{\phi}_{1}\right|^{2} d x+\lambda t^{4} \int_{\Omega}\left[\frac{\left|\tilde{\phi}_{1}\right|^{4}}{4 !}-\frac{t^{2}\left|\tilde{\phi}_{1}\right|^{6}}{6 !}+\cdots\right] d x,
$$

and

$$
J\left(t \phi_{2}\right)=-\frac{t^{2}}{2}\left(\lambda-\tilde{\lambda}_{2}\right) \int_{\Omega}\left|\tilde{\phi}_{2}\right|^{2} d x+\lambda t^{4} \int_{\Omega}\left[\frac{\left|\tilde{\phi}_{2}\right|^{4}}{4 !}-\frac{t^{2}\left|\tilde{\phi}_{2}\right|^{6}}{6 !}+\cdots\right] d x .
$$

Since $\tilde{\phi}_{1}$ and $\tilde{\phi}_{2}$ correspond to different eigenvalues, they are linearly independent in $H_{0}^{1}(\Omega)$. Thus the Morse index of $J(v)$ at $v=0$ is greater than or equal to 2, if $\lambda>\tilde{\lambda}_{2}$. Since $w_{1}$ is the mountain-pass type critical point of $J(v)$, one has that the Morse index of $J(v)$ at $v=w_{1}$ is less than or equal to 1 . Thus $w_{1} \neq 0$ if $\lambda>\lambda_{2}$. Therefore, (3.1) admits four nontrivial solutions: $\pm w_{0}$ and $\pm w_{1}$. By applying the techniques in $[\mathbf{C C N}]$, one can show that $w_{1}$ is sign-changing. Due to the limit of length of this paper, we omit the details.

In fact, upon applying a general theorem (Theorem 9.6) in Rabinowitz [Ra] we can easily check the four assumptions therein are satisfied by (3.1). Thus we have 
Theorem 3.10. If $\lambda>\tilde{\lambda}_{k}$, then (3.1) admits at least $k$ different pairs of nontrivial solutions.

We note that even though our Theorem 3.9 appears to be only a special case of Theorem 3.10, our proof contains slightly more information about the sign-changing solutions than what the approach in $[\mathbf{R a}]$ would provide.

\section{Visualization of positive solutions of the elliptic sine-Gordon equation subject to the homogeneous Dirichlet boundary condition}

In this section, we compute positive solutions by minimizing $J$ on $H_{0}^{1}(\Omega)$ for a dumbbell-shaped domain in 2D. The algorithm (based on the method of steepest descent) for finding the global minimum of $J$ as guaranteed by Theorems 3.3 and 3.4 proceed as follows (assuming $\lambda>\tilde{\lambda}_{1}$ ):

(1) Choose an initial guess $u_{0} \in H_{0}^{1}(\Omega)$ such that

$$
0<u_{0}(x) \leq \pi, \quad J\left(u_{0}\right)<0
$$

(2) Find a $T>0$ such that $J\left(T u_{0}\right) \geq 0$;

(3) Find a $t^{*} \in(0, T)$ such that

$$
J\left(t^{*} u_{0}\right)=\min _{t \in[0, T]} J\left(t u_{0}\right)
$$

(4) Let $u_{0}$ be replaced by $t^{*} u_{0}$. Compute $v_{0}=\nabla J\left(u_{0}\right)$ by

$$
\begin{cases}\Delta v=\Delta u_{0}-\lambda \sin u_{0}, & \text { on } \Omega, \\ v=0, & \text { on } \partial \Omega ;\end{cases}
$$

(5) If $\|v\|<\varepsilon$, then output $u_{0}$; otherwise, solve

$$
s=\arg \min _{s>0} J\left(u_{0}-s v\right),
$$

replace $u_{0}$ by $u_{0}-s v$, and goto Step (3).

The dumbbell-shaped domain $\Omega$ is given in Fig. 2. It is formed by a disk on the left, centered at $(-1,0)$ with radius $1 / 2$, connected through a rectangular corridor with width $1 / 2$, to a disk on the right centered at $(2,0)$ with radius 1 . A boundary element method $[\mathbf{C Z}]$ is used to compute the numerical solution of the linear elliptic BVP (4.1) in Step (4). Five values of $\lambda$ are used:

$$
\lambda=20, \quad 25, \quad 50, \quad 100, \quad \text { and } 500 .
$$

Their graphics are given, in sequential order, in Figs. 3-7, along with the values of $J$ and the maximum value of the function. We see that such solutions' maxima converge to a value close to $\pi=3.1416$. 
14 GOONG CHEN, ZHONGHAI DING, CHIA-REN HU, WEI-MING NI, AND JIANXIN ZHOU

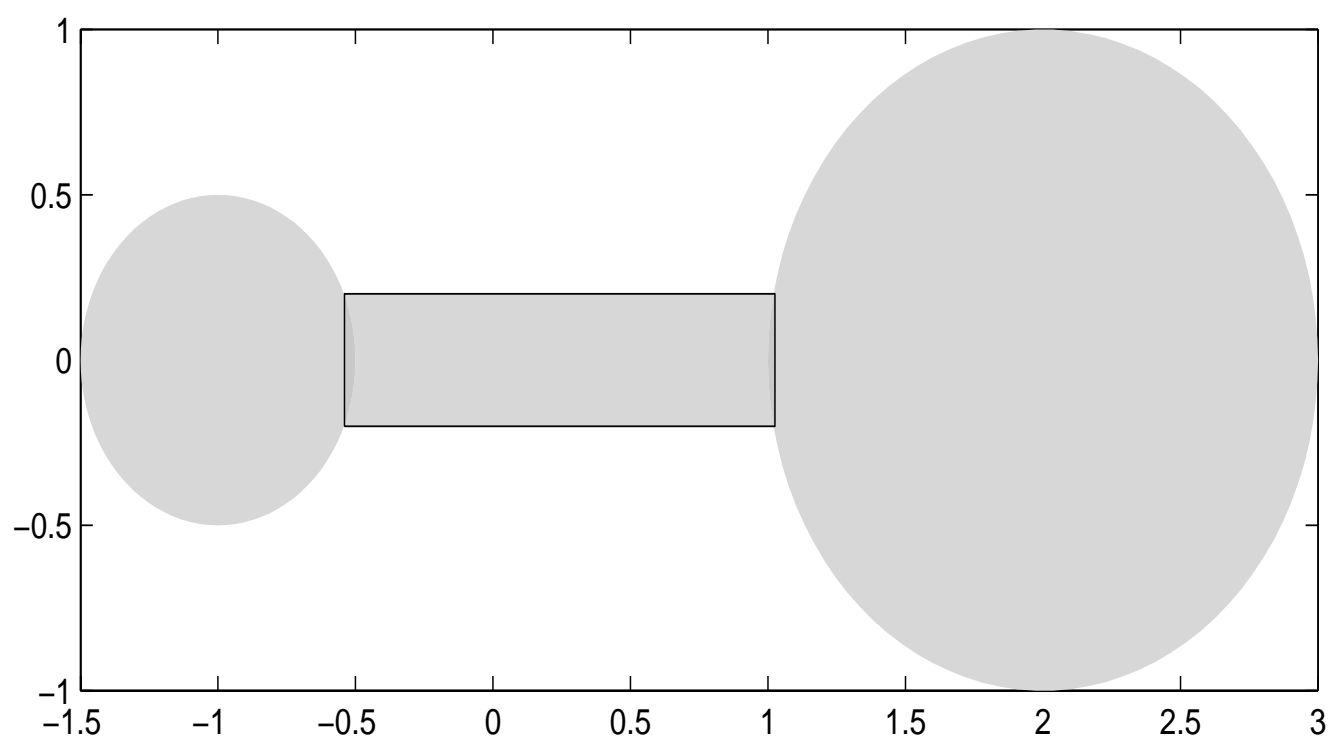

Figure 2. The Dumbbell-shaped domain $\Omega$

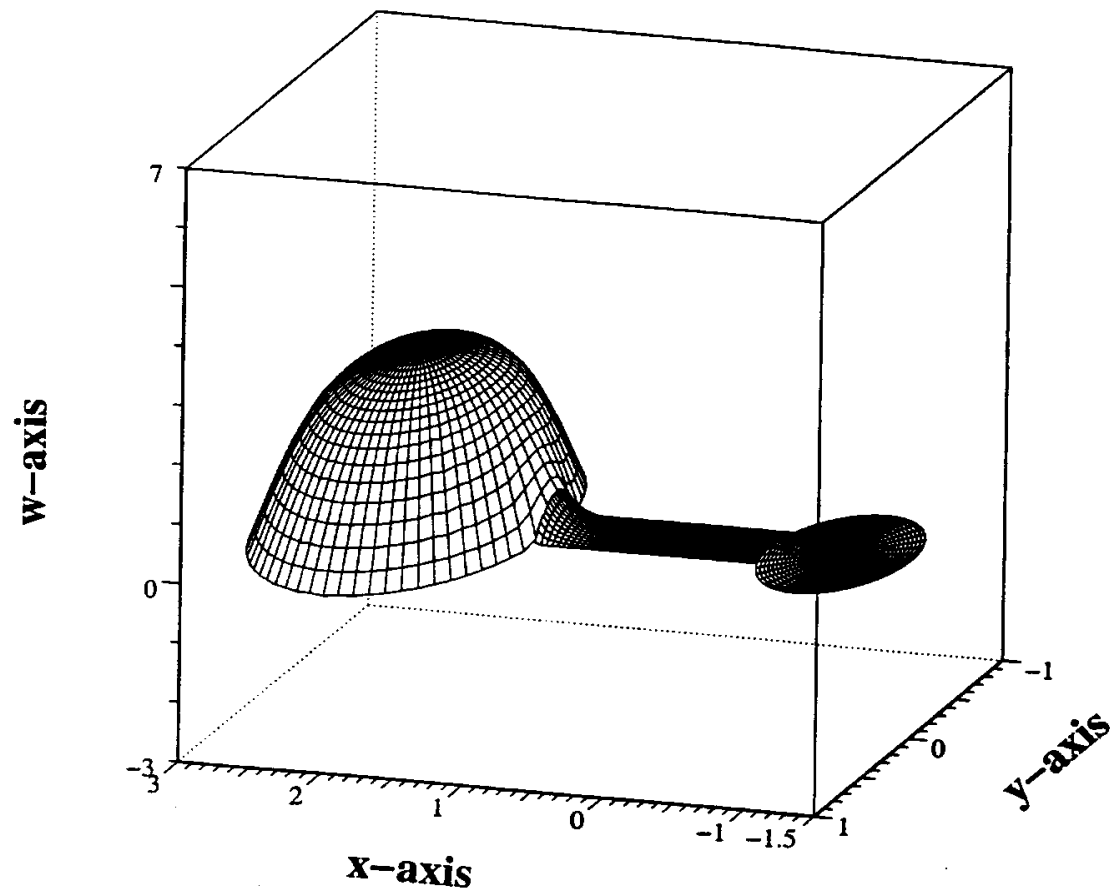

Figure 3. Profile of the global minimizer $w$ for $J_{\lambda}$ on $H_{0}^{1}(\Omega)$, $\lambda=20, J_{\lambda}(w)=-33.47, \max w=2.883$. 


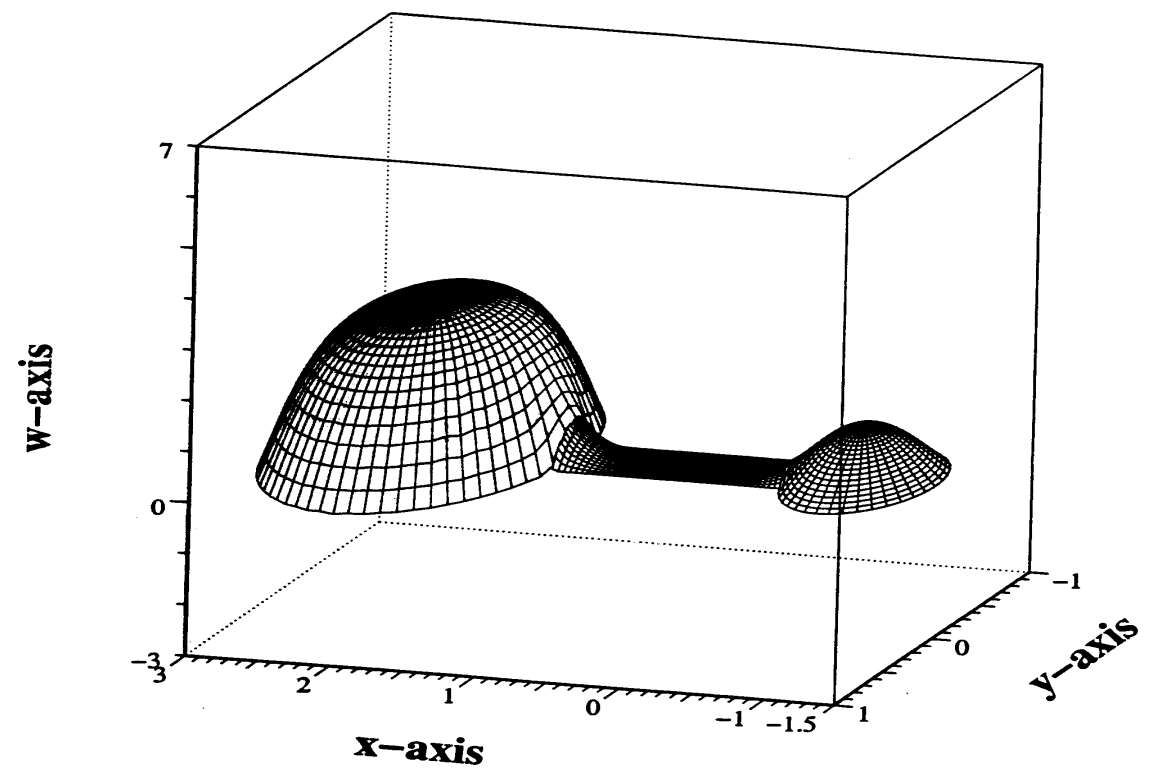

Figure 4. Global minimizer $w, \lambda=25, J_{\lambda}(w)=-51.73, \max w=2.984$.

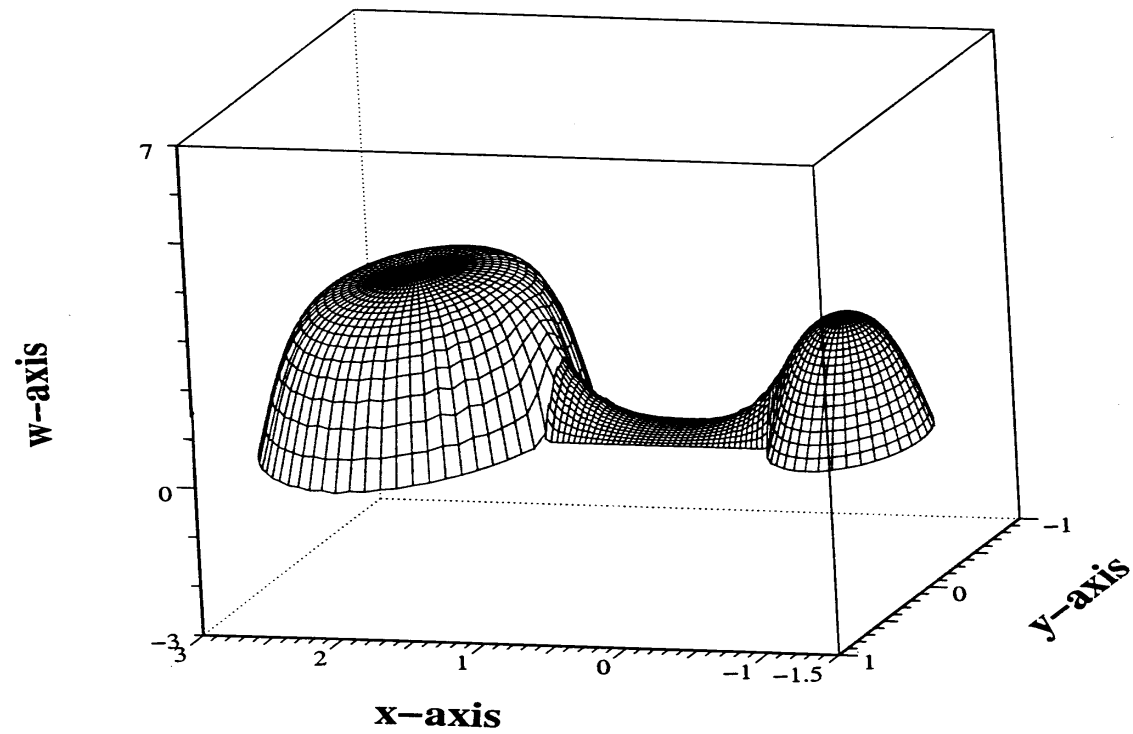

Figure 5. Global minimizer $w, \lambda=50, J_{\lambda}(w)=-169.7, \max w=3.119$. 


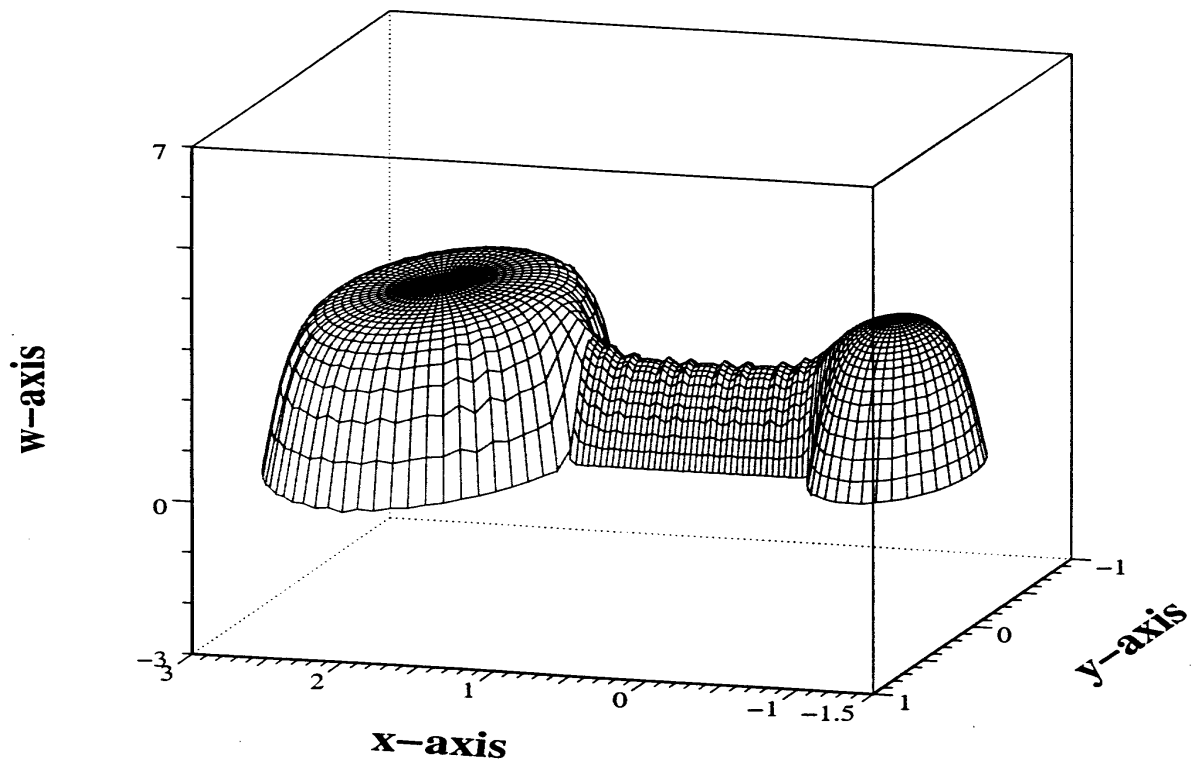

Figure 6. Global minimizer $w, \lambda=100, J_{\lambda}(w)=-469.9$, $\max w=3.14$.

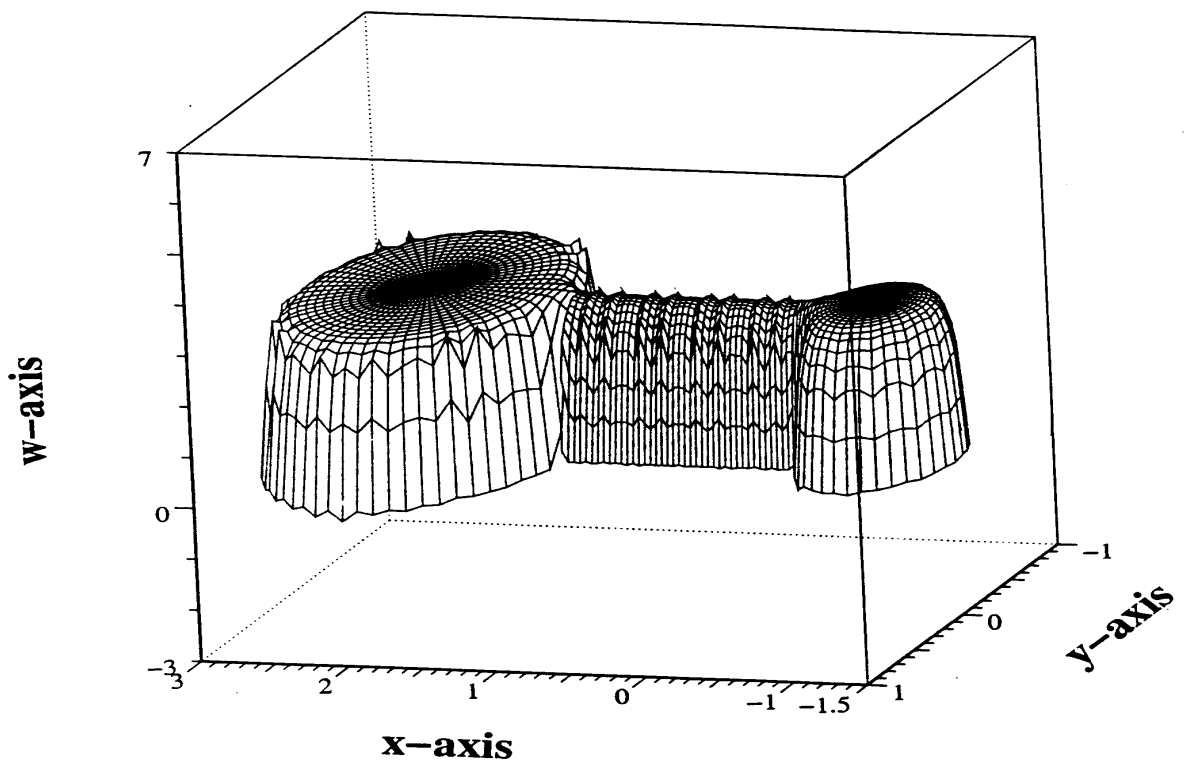

Figure 7. Global minimizer $w, \lambda=500, J_{\lambda}(w)=-3506.7$, $\max w=3.142$. Note that because of the large value of $\lambda$, numerical instability becomes noticeable. 
Acknowledgement. We thank Professor Zhi-Qiang Wang for helpful discussions.

\section{References}

[AH] A. Ambrosetti and P. Hess, J. Math. Anal. Appl., 73, 411 (1980).

[AR] A. Ambrosetti and P. H. Rabinowitz, J. Funct. Anal., 14, 349 (1973).

[CCN] A. Castro, J. Cossio and J. Neuberger, Rocky Mountain J. Math., 27, 1041 (1997).

[Ch] K. C. Chang, Infinite Dimensional Morse Index Theory and Multiple Solution Problems, Birkhäuser, Boston, 1993.

[CZ] G. Chen and J. Zhou, Boundary Element Methods, Academic Press, San Diego, 1992.

[Dr] P. G. Drazin, Solitons, Cambridge Univ. Press, London, 1983, p. 101.

[GNN] B. Gidas, W. M. Ni and L. Nirenberg, Comm. Math. Phys., 68, 209 (1979).

[Ku] I. O. Kulik, Sov. Phys. JETP 1, 251 (1962); T. Kontos et al., Phys. Rev. Lett. 89, 137007 (2002); Y. Blum et al., Phys. Rev. Lett. 89, 187004 (2002).

[La] G. L. Lamb, Jr., Elements of Soliton Theory, John Wiley and Sons, New York, 1980, Sec. 6.4

[Li] C. Li, The existence of infinite many solutions of a class of nonlinear elliptic equations with Neumann boundary condition for both resonance and oscillation problems, preprint, 2002.

[LW] S. J. Li and Z. Q. Wang, J. d'Anal. Math., 81, 373(2000).

[MK] K. Maki and P. Kumar, Phys. Rev. B 14, 3920 (1976), and earlier references cited therein.

[Mi] H. J. Mikeska, J. Phys. C 11, L29 (1978).

[Ra] P. H. Rabinowitz, Minimax methods in critical point theory with applications to differential equations, CBMS 65, AMS, 1986.

[RS] C. Rogers and W. F. Shadwick, Bäcklund Transformations and Their Applications, Academic Press, New York, 1982.

[Sc] A. C. Scott et al., Nuovo Cimento B 69, 241 (1970).

[SR] M. Sigrist and T. M. Rice, J. Phys. Soc. Jpn. 61, 4283 (1992); C. C. Tsuei and J. R. Kirtley, Rev. Mod. Phys. 72, 969 (2000).

Department of Mathematics, Texas A\&M University, College Station, TX 77843

E-mail address: gchen@math.tamu.edu

Department of Mathematical Sciences, University of Nevada, 4505 Maryland ParkWAY, LAS VEGas, NV 89154-4020

E-mail address: dingz@unlv.edu

Department of Physics, Texas A\&M University, College Station, TX 77843

E-mail address: hu@rainbow.physics.tamu.edu

School of Mathematics, University of Minnesota, Minneapolis, MN 55455

E-mail address: ni@math.umn.edu

Department of Mathematics, Texas A\&M University, College Station, TX 77843

E-mail address: jzhou@math.tamu.edu 